\title{
Therapeutic Use of Stem Cell Transplantation for Cell Replacement or Cytoprotective Effect of Microvesicle Released from Mesenchymal Stem Cell
}

\author{
Moonhwan Choi ${ }^{1}$, Taehyun Ban ${ }^{1}$, and Taiyoun Rhim ${ }^{1,2, *}$
}

Idiopathic pulmonary fibrosis (IPF) is the most common and severe type of idiopathic interstitial pneumonias (IIP), and which is currently no method was developed to restore normal structure and function. There are several reports on therapeutic effects of adult stem cell transplantations in animal models of pulmonary fibrosis. However, little is known about how mesenchymal stem cell (MSC) can repair the IPF. In this study, we try to provide the evidence to show that transplanted mesenchymal stem cells directly replace fibrosis with normal lung cells using IPF model mice. As results, transplanted MSC successfully integrated and differentiated into type II lung cell which express surfactant protein. In the other hand, we examine the therapeutic effects of microvesicle treatment, which were released from mesenchymal stem cells. Though the therapeutic effects of MV treatment is less than that of MSC treatment, MV treat-ment meaningfully reduced the symptom of IPF, such as collagen deposition and inflammation. These data suggest that stem cell transplantation may be an effective strategy for the treatment of pulmonary fibrosis via replacement and cytoprotective effect of microvesicle released from MSCs.

\section{INTRODUCTION}

Pulmonary fibrosis occurs as a wound-healing process after many forms of pulmonary injury that is induced by a variety of factors. However, chronic inflammation and pulmonary fibrosis can be developed without any known causes in certain cases. That is classified as idiopathic interstitial pneumonias (IIPS) (Steele and Schwartz, 2012). Idiopathic pulmonary fibrosis (IPF) is the most common type of IIP (Raghu et al., 2011; Steele and

${ }^{1}$ Department of Bioengineering, College of Engineering, Hanyang University, Seoul 133-791, Korea, ${ }^{2}$ Present address: Institute for Bioengineering and Biopharmaceutical Research, Hanyang University, Seoul 133-791, Korea

*Correspondence: rhim@ hanyang.ac.kr
Schwartz, 2012), and is considered to be the most severe form of pulmonary fibrosis, with a $50 \%$ mortality rate three years after diagnosis (King et al., 2001).

Until 2009, no approved medication to treat IPF existed. Recently, pirfenidone was approved for the treatment of patients with IPF in Japan (Luppi et al., 2012) for its effectiveness on suppressing fibrosis via inhibition of TGF- $\beta$-induced collagen synthesis (lyer et al., 1999). Although pirfenidone had a therapeutic effect on lung functional deterioration and disease progression in patients with IPF (Taniguchi et al., 2010), it could not regenerate the damaged tissue.

To regenerate the damaged tissues, stem cell therapy has been tried for IPF. The different studies have shown that adult stem cells contribute to preventing fibrosis progression, repair and remodeling of lung in animal models of pulmonary fibrosis (Banerjee et al., 2012; Lee et al., 2010). The first and the most important suggested mechanism of stem cell therapy is a cell replacement - replacement of damaged cells by brand new stem cells. However, they showed us only existences of transplanted stem cells in recipient tissue by labeling stem cells (Zhao et al., 2008) sex specific genes or by male-specific markers such as Sry (Zhao et al., 2013) not direct evidences of replacement. Even more, the numbers of transplanted stem cells were rather small to change the function of whole tissue.

More recently, there is increasing experimental evidence for re-program injured cells to repair damaged tissues by the materials, which were released from transplanted stem cells not by themselves. Microvesicle released from mesenchymal stem cells, resulting in a horizontal transfer of mRNA, microRNA and proteins which can regulated the target cells (Hu et al., 2012). The transfer of gene products from stem cells may explain the therapeutic effects of stem cell transplantation without the need of trans-differentiation into tissue cells (Camussi et al., 2010).

In this respect, we try to examine the two possible mechanisms of pulmonary function restoration after human mesenchymal stem cell (hMSCs) transplantation by examining direct differentiation of transferred hMSCs into lung cell using human sequence specific RT-PCR and checking the effect of stem cell-derived microvesicles. 


\section{MATERIALS AND METHODS}

\section{Human mesenchymal stem cells and microvesicle} preparation

Bone marrow-derived human mesenchymal stem cells (hMSCs) were purchased from Lonza (Switzerland) and cultured in high glucose Dulbecco's modified Eagle's medium (DMEM) containing $20 \%$ fetal bovine serum (Gibco, Invitrogen, USA) at $37^{\circ} \mathrm{C}$ in a $5 \% \mathrm{CO}_{2}$ atmosphere (Szade et al., 2011).

Microvesicles were isolated from conditioned media of hMSCs by using the ExoQuick (SBI Biosciences, USA). Briefly, $10 \mathrm{ml}$ of cultured medium (centrifuged at $5,000 \times g$ for $5 \mathrm{~min}$ ) was mixed $2 \mathrm{ml}$ of ExoQuick precipitation solution and incubated overnight at $4^{\circ} \mathrm{C}$. After incubation, exosomes were precipitated at 5,000 $\times g$ for $30 \mathrm{~min}$. Pellets were washed with phosphate buffered saline (PBS), and concentrated with centrifugation.

\section{Construction of mice fibrosis model}

Female BL/6J mice (5 weeks old) were purchased from Japan SLC, Inc. (Japan) and randomly divided into two fibrosis groups and a normal control group. First fibrosis group received $200 \mu \mathrm{g}$ of silica per $\mathrm{kg}$ of body weight by intratracheal injection.

Mice receiving silica group were then injected with hMSCs $(2$ $\times 10^{5}$ cells in $100 \mu \mathrm{l}$ PBS) or microvesicle (10 $\mu \mathrm{g}$ in $100 \mu \mathrm{l}$ PBS) released from hMSCs via the tail vein under anesthesia at 12 weeks and 14 weeks (Fig. 1). All the mice were weighed once a week. All care and experimental procedures for the mice were performed under the guidelines of Hanyang University Policy for Experiments in Animals, and all protocols were approved by the IACUC of the Hanyang University.

\section{Wet/dry ratio determination}

After the animals were sacrificed, the right upper bronchus lobe was tied with a string. Following BAL of the right lung, right lungs were removed for histological analysis. The left lung lobes were removed and then placed in a desiccant in an oven at $60^{\circ} \mathrm{C}$ and re-weighed after $24 \mathrm{~h}$. Dry weights were recorded when they remained constant on successive days.

\section{Bronchial alveolar lavage procedure}

The BAL fluid was recovered from each animal and BAL cells were collected using clinical centrifugation. Cytocentrifuge slides of BAL cells were fixed in methanol, and stained with Diff-Quik. Five hundred leukocytes were counted and foamy macrophages were enumerated as described previously (Bedrossian et al., 1991).

\section{Histological analysis}

Lungs used for histology were inflation fixed with $10 \%(\mathrm{v} / \mathrm{v})$ buffered formaldehyde, dehydrated with a series of graded ethanol, and embedded in paraffin. Specimens were sliced into 4- $\mu$ m-thick sections and stained with H\&E or Masson's trichrome to evaluate collagen production. Microscopic examination was performed and photographed under a regular light microscope.

Fibrosis volume was quantified with Masson's trichrome staining as the area stained positive for collagen. Fibrotic and non-fibrotic areas were calculated by integrating ten fibrosis section volumes, where fibrosis volume was defined as (blue area/total area) $\times$ (weight of each section/total weight of 10 sections) $\times 100 \%$.
Nested RT-PCR (reverse transcription-PCR) and sequencing

Total RNA was isolated from liver tissue using TRIzol reagent (Invitrogen, USA) according to the manufacturer's instructions. The cDNA was prepared using a Superscript III kit (Invitrogen) according to the manufacturer's instructions. The human surfactant protein B (SFTPB) CDNA was amplified by PCR (30 s $94^{\circ} \mathrm{C}, 45 \mathrm{sec}$ at $66^{\circ} \mathrm{C}$, and $45 \mathrm{~s}$ at $72^{\circ} \mathrm{C}$ for 35 cycles) using the $1^{\text {st }}$ hSFTPB forward primes and $1^{\text {st }}$ hSFTPB reverse primers d(GCC CCG ACC TTT GAT GAG AA) and d(GCA GTG GCT GGT TTT TCC TG), respectively, which amplify a 371 bp sequence, followed by a second PCR with the $2^{\text {nd }}$ hSFTPB forward primes and $2^{\text {nd }}$ hSFTPB reverse primers d(AAA GAA GCC TCA GCT CCC ACA CCG) and d(AGG CTG CAG AGC TCC TCC AGA TG) nested primers, respectively, which amplify a $195 \mathrm{bp}$ sequence. The primers used for PCR amplifying $\mathrm{m} \beta$ actin mRNA were d(GGA CTC CTA TGT GGG TGA CGA GG) and d(GGG AGA GCA TAG CCC TCG TAG AT).

Amplified DNAs were purified by using PCR/Gel Combo kit (NucleoGen, Korea). And the DNA sequencing was performed at Genotech (Korea). The DNA sequences were compared and aligned using ClustalW 2.0 program (www.ebi.ac.uk/clustalw).

\section{Western blot}

Protein extracts were obtained from liver tissue and lysed by sonication in PBS containing $1 \mathrm{mM}$ PMSF and protease inhibitor cocktail (Sigma-Aldrich, USA). Proteins were resolved by $12.5 \%$ SDS-PAGE and transferred to a PVDF membrane (Millipore, Italy). The membrane was blocked for $1 \mathrm{~h}$ in a $5 \%$ skim milk solution and then incubated with polyclonal rabbit antimouse collagen type I antibody (1:500 dilution) (Chemicon, USA) or polyclonal rabbit anti-mouse SMA- $\alpha$ antibody (1:1000 dilution) (Abcam, USA) for $2 \mathrm{~h}$ at room temperature, respectively. The unbound primary antibodies were removed with one 15 min and two 5 min washes in PBS containing $0.1 \%(\mathrm{v} / \mathrm{v})$ Nonidet $\mathrm{P}-40$. Then, the membrane was incubated with horseradish peroxidase-conjugated goat anti-mouse IgG secondary antibody (Ab Frontier, Korea) for $2 \mathrm{~h}$ at room temperature. The target protein was detected by ECL solution (Amersham Biosciences, USA) using X-ray film.

\section{Human specific nuclear antigen detection}

Sections of the fixed embedded tissues were cut to $4 \mu \mathrm{m}$ thickness, and on placed onglass slides. The sections were deparaffinized and rehydrated. The sections were incubated in blocking buffer for $1 \mathrm{~h}$ at room temperature to block non-specific binding. The anti-human nuclei monoclonal antibody (1: 100 dilution) (Millipore, Italy) was used as a primary antibody. Sections were incubated overnight at $4^{\circ} \mathrm{C}$. Bound primary antibody was detected by addition of FITC conjugated mouse IgG secondary antibody (1:32 dilution) (Ab frontier, Korea) for $1 \mathrm{~h}$ at room temperature. 6-diamidino-2-phenylindole (DAPI) (USB, USA) was used to stain the nuclei.

\section{Statistical analysis}

All quantitative data are expressed as mean \pm standard deviation. Statistical analysis was performed with one-way analysis of variance (ANOVA) using SPSS software (SPSS Inc., USA). A value of $p<0.05$ was considered statistically significant.

\section{RESULTS}

Wet/dry weight changes

To measure the pulmonary edema, we determined the wet/dry 


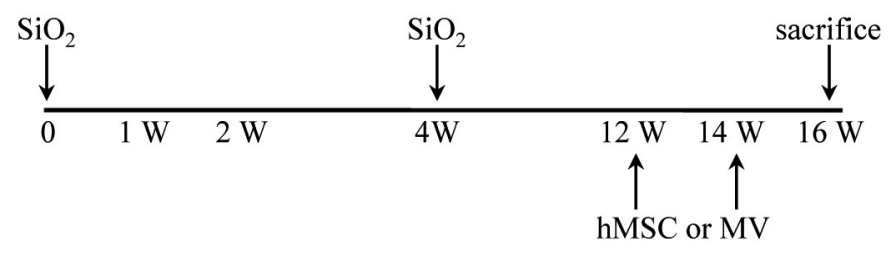

Fig. 1. Schematic representation of silica induced pulmonary fibrosis model with hMSC or MV treatment. The $\mathrm{SiO}_{2}-$ induced IPF mice model was treated with hMSC or MV. IT, intratracheal; IV, intravein treated.

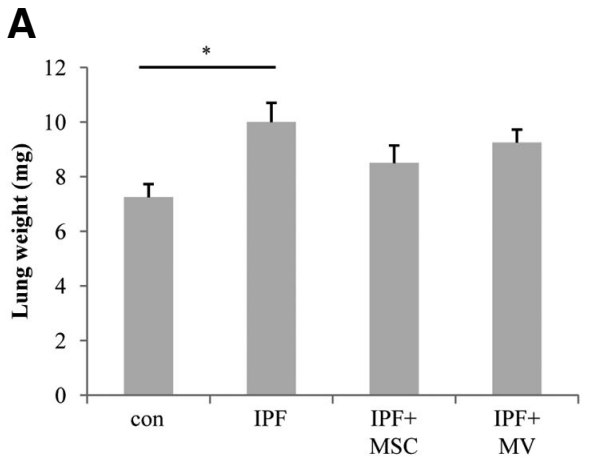

A

weight of whole lungs. After 16 weeks of silica treatment, the mice were sacrificed and tissues removed. Figure 2 shows edema formation in idiopathic pulmonary fibrosis as indicated by increased silica treated lung weight and an increase in wetto-dry weight ratio. Silica treated mice showed significantly higher lung lobe weight and wet-to-dry weights of lung than sham-treated mice (Fig. 2, $p=0.018, p=0.003$, respectively). There is no significance of lung weight among silica treated mice, hMSC and microvesicle treated mice (Fig. 2A). But, the transplantation of hMSCs into silica treated mice reduced the wet/dry ratio meaningfully (Fig. $2 B, p=0.049$ ). For microvesicle transplanted group was a tendency, though not significant, of decreased wet/dry ratio (Fig. $2 B, p=0.064$ ).

\section{Effects hMSC or microvesicle treatment on BAL cells in silica induced fibrosis model mice}

After 16 weeks of silica treatment, BAL cells were collected, cytospin onto slides, and stained with Diff-Quick for determination of cellular differentials. The lung tissues were stained with H\&E and Masson's trichrome to evaluate histopathologic changes and tissue fibrosis.

Numbers of inflammatory cells such as macrophage (Fig. 3A), Neutrophils (Fig. 3B) and lymphocytes (Fig. 3C) were significantly increased in silica treated fibrosis model. Treatment of both hMSCs or microvesicle released from hMSC meaningfully decreases the numbers of total BAL cells as well as neutrophils and lymphocytes in silica induced fibrosis model mice. Treatment of hMSC or microvesicle not only decreased the numbers of BAL cells but also they decreased the percentage of foamy macrophages among the total macrophages (Fig. 3D). Previously, we had reported that the percentage of foamy macrophages among the total macrophages in the BAL fluid was directly associated with extend of fibrosis and the concentration of apoA1 in BAL fluid (Kim et al., 2010). As shown in Figs. 3D and 4 , numbers of foamy macrophages were increased as other fibrosis model, however treatment of both hMSCs and microvesicle derived from hMSC decreased the numbers of foamy macrophage.
Reduction of fibrosis by hMSCs or Microvesicle treatment The degree of lung fibrosis was evaluated histologically using Masson's trichrome staining. Fibrosis developed in the lung parenchyma of silica treated mice. However, 12 weeks and 14 weeks after hMSC treatment, the degree of inflammation and Masson's stained area and density were decreased (Fig. 5B) Also, treatment of microvesicle decreased the Masson's trichrome staining area. For quantification of histological data, we examined the tissue slides from all lung sections, and the results are expressed as percentages. As shown in Fig. $5 \mathrm{C}$, the area occupied by blue-stained collagen was decreased significantly by both hMSC transplantation $(60.7 \%$ vs. $39.4 \%, p=$ $0.047)$ and microvesicle treatment $(60.7 \%$ vs. $55.5 \%)$.

The amounts of collagen I and SMA- $\alpha$, which was mainly deposited in fibrotic area of lung (Liu et al., 2007; Selman et al., 2001), were measured by Western blot analysis of lung crude extracts. As shown in Fig. 6A, amount of collagen I was increased in fibrosis model however, it was meaningfully decreased after hMSCs transplantation or microvesicle treatment. On the other hand, amount of SMA- $\alpha$ was only significantly decreased in hMSCs treated group (Fig. 6B).

Detection of differentiated hMSCs in mouse lung tissues To detect hMSCs in transplanted mice lung, we used human nuclear antigen specific antibody. As shown in Fig. 7A, human originated cells were detected (arrow indicated) in lung tissues. These data were well matched with our previous report ( $\mathrm{Ha}$ et al., 2012). To examine whether transplanted hMSCs developed into functionally active lung cells or not, we conducted RT-PCR. Total RNAs were extracted from lung tissue and reverse transcribed with oligo dT primers. As shown in Fig. 7B, the gene expression of human surfactant protein B (hSFTPB), a marker of differentiated lung II cells (Guttentag et al., 1998), was found only in hMSC transplanted mouse lung according to RT-PCR. The amplified PCR products showed $100 \%$ sequence homology to the human SFTPB (Fig. 7C). In addition, the primers that we used in RT-PCR were specific to different exons within the human SFTPB gene. These results suggest that transplanted 

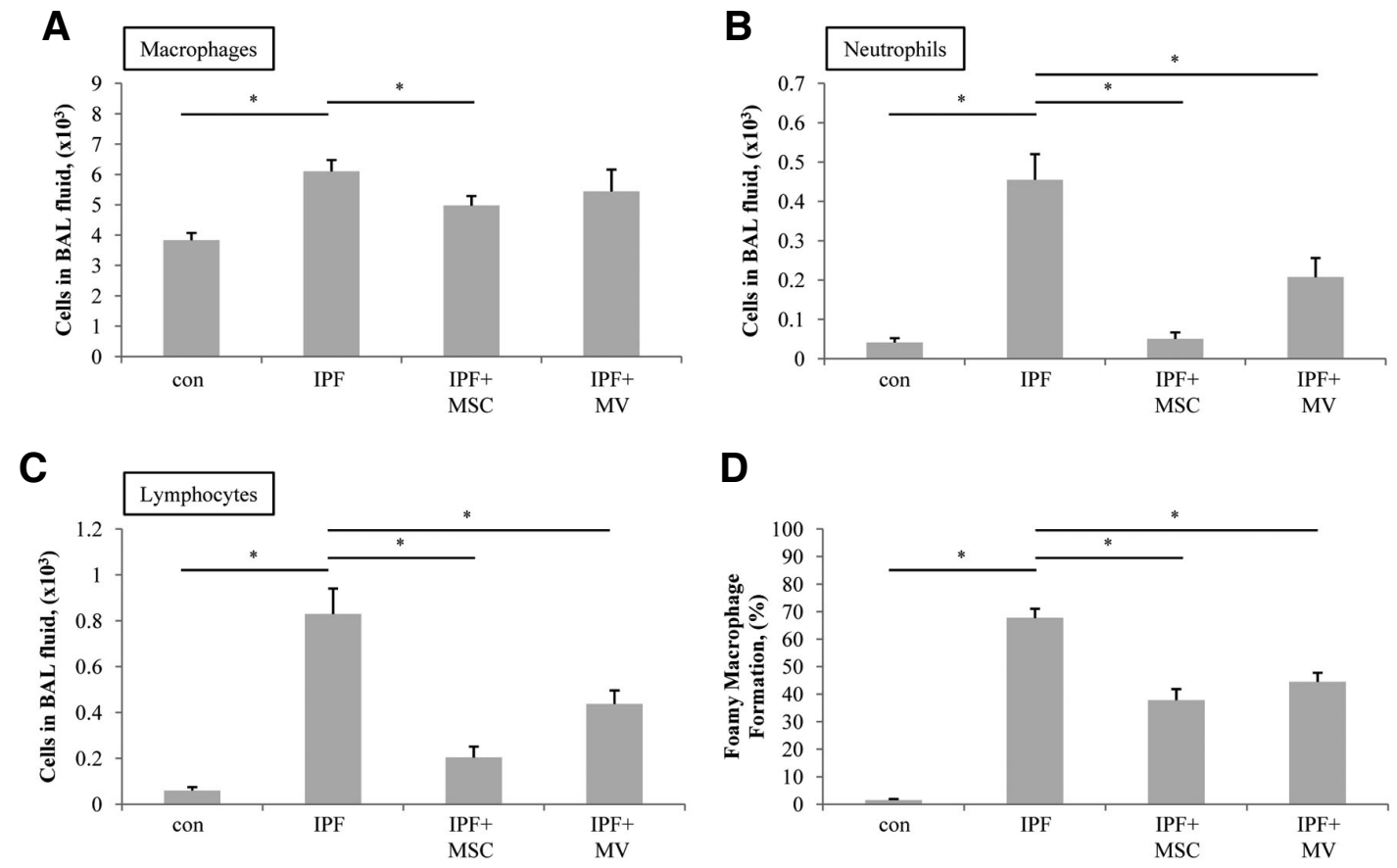

Fig. 3. The inflammatory cell distribution in BAL fluid of pulmonary fibrosis model mice with $\mathrm{hMSC}$ or MV treatment. BAL fluids were collected and analyzed for the numbers of macrophages (A), neutrophils $(B)$ and lymphocytes $(C)$. The number of neutrophils, lymphocytes, and macrophages in the BAL fluid increased in pulmonary fibrosis model and treatment of hMSCs or MV reduced the number of inflammatory cells in BAL fluid. Treatment of hMSCs or MV decrease the percentage of foamy macrophages among total alveolar macrophages (D).
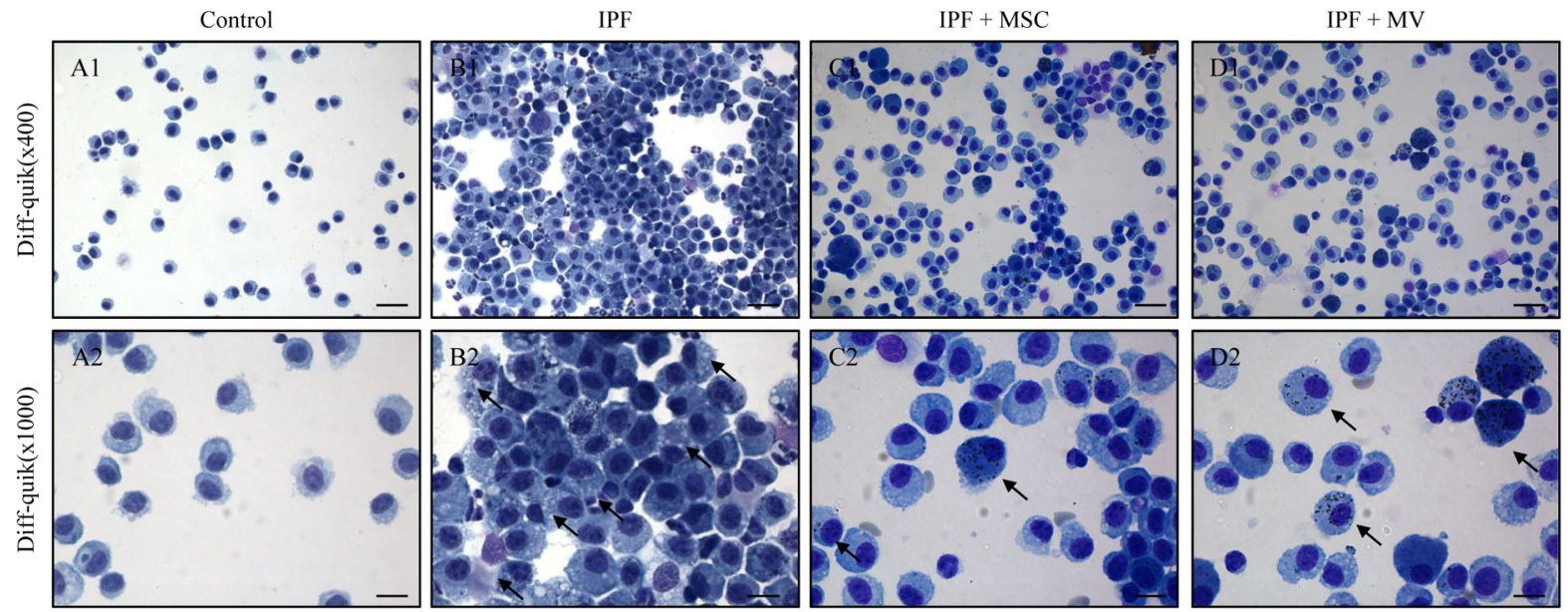

Fig. 4. Diff-quick staining of BAL cells. Numbers of total BAL cells were increased in silica induced fibrosis model (B), however treatment of hMSC or MV driven hMSC significantly decreased the BAL cells in the airway $(C, D)$. Foamy macrophages were indicated with arrow head.

hMSCs directly differentiate into functional lung I cells.

\section{DISCUSSION}

Stem cells have a potential to be differentiated into special tissues, so they have attract attention as a candidate therapy for degenerative diseases. Idiopathic pulmonary fibrosis is a good example of degenerative disease, which is currently no method was developed to restore normal structure and function.
In fact, there are several reports on therapeutic effect of various stem cell transplatation on pulmonary fibrosis model (Ortiz et al., 2003). They mainly used bleomycin induced fibrosis model. There are several methods to produce an animal model for pulmonary fibrosis animal model including exposure to bleomycin, fluorescein isothiocyanate (FITC) or silica; irradiation; or expression of specific genes through delivery of a viral vector or utilization of a transgenic system (Moore and Hogaboam, 2008). Though intratrachea bleomycin model is well 
A

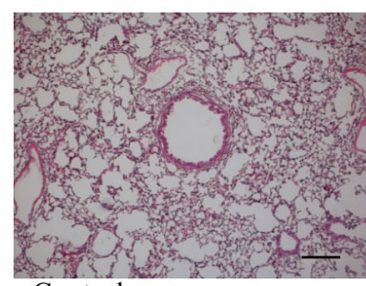

Control

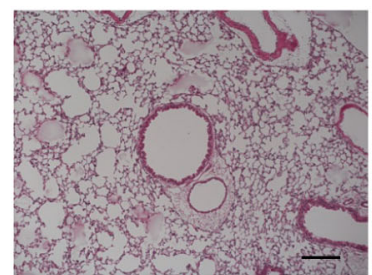

$\mathrm{IPF}+\mathrm{MSC}$
HE stain(x100)

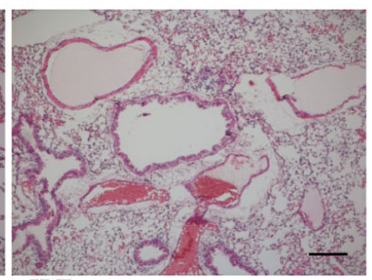

IPF

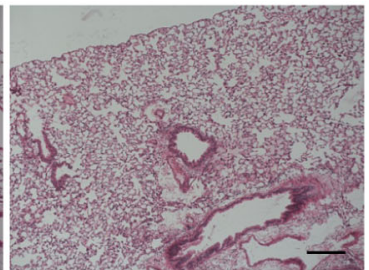

$\mathrm{IPF}+\mathrm{MV}$
B
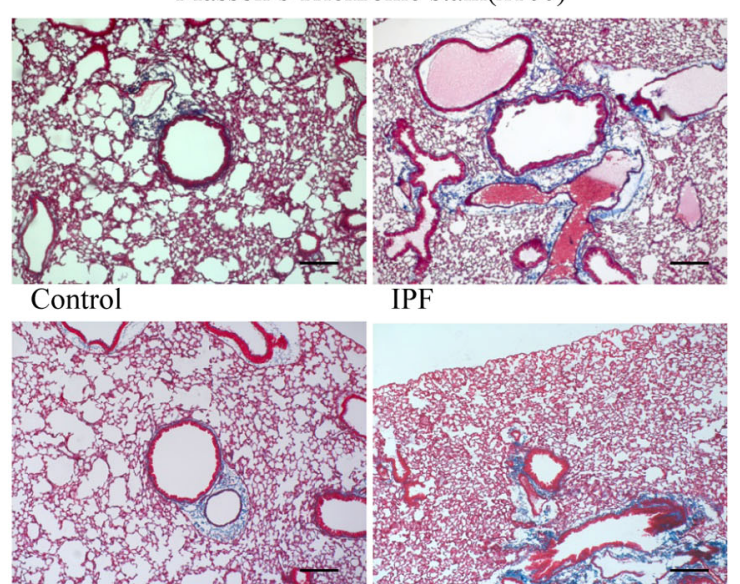

$\mathrm{IPF}+\mathrm{MSC}$
IPF

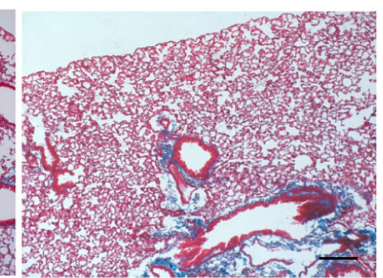

$\mathrm{IPF}+\mathrm{MV}$

C

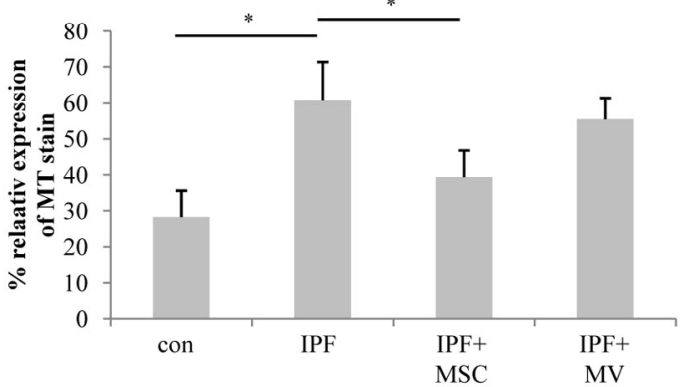

Fig. 5. Effect of hMSCs or microvesicle transfer on collagen deposition in lung tissues. Collagen deposition in the lungs was analyzed using Masson's Trichrome stain (B). Stained areas were quantified using NIH Image J software (http: //rsb.info.nih.gov/ij/). (C) * ${ }^{*}<0.05$ compared with the fibrosis group.

A

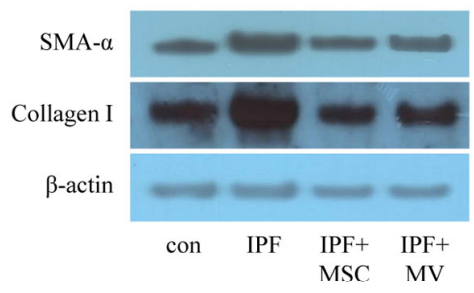

B

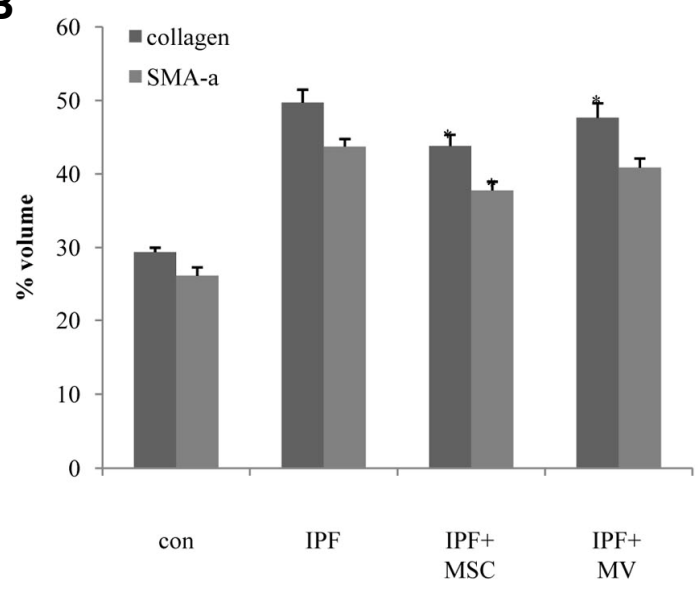

Fig. 6. Collagen I and SMA- $\alpha$ expression following hMSCs or MV treatment. Concentrations of type I collagen and Smooth muscle actin- $\alpha$ were measured by Western blot analysis (A) followed by densitometric quantification (B). characterized model for pulmonary fibrosis, however, this model has a limitations; lacks of fibroblastic foci, hyperplastic epithelium, and temporal heterogeneity. Most of all, pathological findings of bleomycine model look like an acute lung injury model than a fibrosis model. In this point of view, we choose silica induced fibrosis model to compensate the weak point of bleomycin model. Though silica induced fibrosis model has some limitations but validation of stem cell therapy potential using another model is quite important.

As in results section, silica induced fibrosis models were successfully constructed in Wet/dry ratio, BAL cell numbers and morphology and tissue histology. And transplantation of hMSCs significantly reduced every aspects of pulmonary fibrosis as above. Recently, we had reported that transplantation of hMSCs 
A

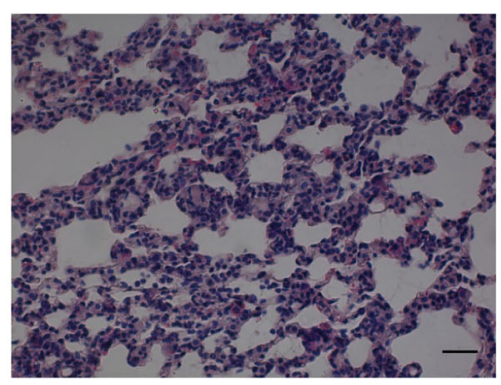

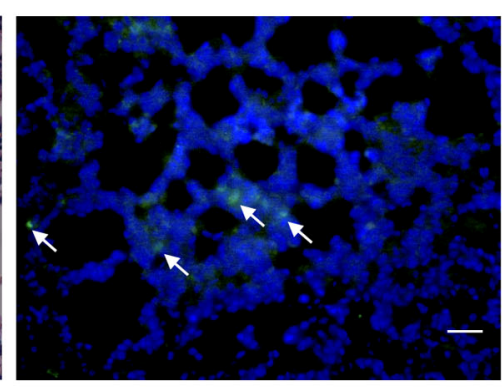

B

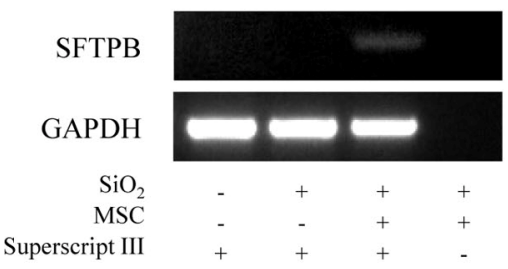

C

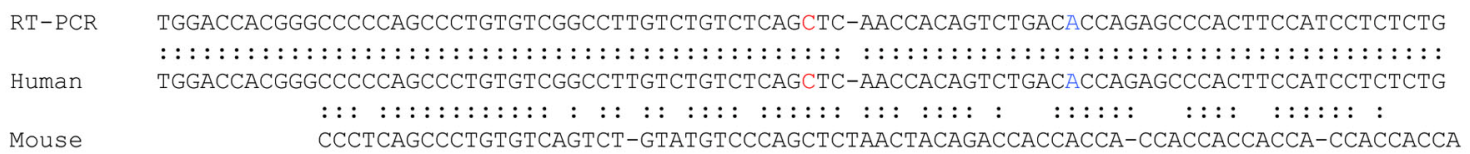

Fig. 7. The Detection of functionally differentiated hMSCs in mouse lung. Representative images of H\&E staining immunofluorescent staining for human-specific nuclear antigen (HuNu, green) (A). Detection of lung-specific human sttpb (surfactant protein B) expression by RT-PCR (B). Amplified cDNA sequence was determined and compared with mouse (NM_001282071) and human SFTPB (NM_000542) cDNA sequences.

showed us a good therapeutic effect on liver fibrosis and also, we had succeeded to find the direct evidence of development of hMSCs into functionally active hepatocytes (Ha et al., 2012). At that time, we had used cy5.5 labeled SPIO molecule to stain hMSCs and chased the transplanted hMSCs. Labeled hMSCs distributed in all internal organ, however, relatively large stained population was found in the lung. In the silica induced pulmonary fibrosis model mice, relatively large amount of hMSCs were found in recipient lung tissue (Fig. 7A) as our previous data. Sequence analyses of amplified DNA sequence by RTPCR revealed that human SFTPB gene was expressed in mice lung. These data suggested that transplanted hMSCs successfully incorporated and developed as functional lung cells.

In the other hand, we examined the therapeutic potential of microvesicle or sometimes called microvesicle from hMSCs. As described in introduction session, series of reports on the therapeutic effects of microvesicle released from stem cells transferring genetic or non-genetic material (Akao et al., 2011). We observed the microvesicle released from hMSCs had therapeutic effects on silica induced pulmonary fibrosis without transferring hMSCs. Microvesicle alone, it reduced the recruitment of inflammatory cells into airways (Figs. 3 and 4), collagen deposition in lung parenchymal (Figs. 5 and 6).

In conclusion, the systemic administration of hMCSs effectively reduced the airway inflammation and collagen deposition that is typically observed in silica induced pulmonary fibrosis models. They were properly incorporated and developed as lung tissue. However, microvesicle released from hMSCs also reduced silica induced pulmonary fibrosis. These data suggest that stem cell transplantation may be an effective strategy for the treatment of pulmonary fibrosis via modulation of microenvironment of injured lung by mechanism of direct substitution and this novel role of microvesicle highlights a new perspective into intercellular mediation of tissue injury and repair, and engenders novel approaches to the development of biologics for tissue repair.

\section{ACKNOWLEDGMENTS}

This study was supported by a grant of the Korean Health Technology R\&D Project, Ministry of Health \& Welfare, Republic of Korea (HI11C0194). Moonhwan choi was partly supported by NRF-2012M3A9D1054451.

\section{REFERENCES}

Akao, Y., lio, A., Itoh, T., Noguchi, S., Itoh, Y., Ohtsuki, Y., and Naoe, T. (2011). Microvesicle-mediated RNA molecule delivery system using monocytes/macrophages. Mol. Ther. 19, 395-399.

Banerjee, E.R., Laflamme, M.A., Papayannopoulou, T., Kahn, M., Murry, C.E., and Henderson, W.R., Jr. (2012). Human embryonic stem cells differentiated to lung lineage-specific cells ameliorate pulmonary fibrosis in a xenograft transplant mouse model. PLoS One 7, e33165.

Bedrossian, C., Warren, C., Ohar, J., and Jackson, F. (1991). Amiodarone pulmonary toxicity - cytopathology ultrastructure and immunocytochemistry. Lab. Invest. 64, A113-A113.

Camussi, G., Deregibus, M.C., Bruno, S., Cantaluppi, V., and Biancone, L. (2010). Exosomes/microvesicles as a mechanism of cell-to-cell communication. Kidney Int. 78, 838-848.

Guttentag, S.H., Beers, M.F., Bieler, B.M., and Ballard, P.L. (1998). Surfactant protein B processing in human fetal lung. Am. J. Physiol. 275, L559-566.

Ha, Y., Shin, J.S., Lee, D.Y., and Rhim, T. (2012). Fluorescently labeled nanoparticles enable the detection of stem cell-derived hepatocytes. B Korean Chem. Soc. 33, 1983-1988.

Hu, G., Drescher, K.M., and Chen, X.M. (2012). Exosomal miRNAs: biological properties and therapeutic potential. Front. Genet. 3, 56.

lyer, S.N., Gurujeyalakshmi, G., and Giri, S.N. (1999). Effects of pirfenidone on transforming growth factor-beta gene expression at the transcriptional level in bleomycin hamster model of lung fibrosis. J. Pharmacol. Exp. Ther. 291, 367-373.

Kim, T.H., Lee, Y.H., Kim, K.H., Lee, S.H., Cha, J.Y., Shin, E.K., Jung, S., Jang, A.S., Park, S.W., Uh, S.T., et al. (2010). Role of lung apolipoprotein $A-I$ in idiopathic pulmonary fibrosis: antiinflammatory and antifibrotic effect on experimental lung injury and fibrosis. Am. J. Respir. Crit. Care Med. 182, 633-642.

King, T.E., Jr., Tooze, J.A., Schwarz, M.I., Brown, K.R., and Cherniack, R.M. (2001). Predicting survival in idiopathic pulmonary fibrosis: scoring system and survival model. Am. J. Respir. Crit. Care Med. 164, 1171-1181.

Lee, S.H., Jang, A.S., Kim, Y.E., Cha, J.Y., Kim, T.H., Jung, S., 
Park, S.K., Lee, Y.K., Won, J.H., Kim, Y.H., et al. (2010). Modulation of cytokine and nitric oxide by mesenchymal stem cell transfer in lung injury/fibrosis. Respir. Res. 11, 16.

Liu, T.J., Chung, M.J., Ullenbruch, M., Yu, H.F., Jin, H., Hu, B., Choi, Y.Y., Ishikawa, F., and Phan, S.H. (2007). Telomerase activity is required for bleomycin-induced pulmonary fibrosis in mice. $\mathrm{J}$. Clin. Invest. 117, 3800-3809.

Luppi, F., Spagnolo, P., Cerri, S., and Richeldi, L. (2012). The big clinical trials in idiopathic pulmonary fibrosis. Curr. Opin. Pulm. Med. 18, 428-432.

Moore, B.B., and Hogaboam, C.M. (2008). Murine models of pulmonary fibrosis. Am. J. Physiol. Lung C 294, L152-L160.

Ortiz, L.A., Gambelli, F., McBride, C., Gaupp, D., Baddoo, M. Kaminski, N., and Phinney, D.G. (2003). Mesenchymal stem cell engraftment in lung is enhanced in response to bleomycin exposure and ameliorates its fibrotic effects. Proc. Natl. Acad. Sci. USA 100, 8407-8411.

Raghu, G., Collard, H.R., Egan, J.J., Martinez, F.J., Behr, J., Brown, K.K., Colby, T.V., Cordier, J.F., Flaherty, K.R., Lasky, J.A., et al. (2011). An official ATS/ERS/JRS/ALAT statement: idiopathic pulmonary fibrosis: evidence-based guidelines for diagnosis and management. Am. J. Respir. Crit. Care Med. 183, 788-824.

Selman, M., King, T.E., Pardo, A., American Thoracic, S., European Respiratory, S., and American College of Chest, P. (2001). Idio- pathic pulmonary fibrosis: prevailing and evolving hypotheses about its pathogenesis and implications for therapy. Ann. Int. Med. 134, 136-151.

Steele, M.P., and Schwartz, D.A. (2012). Molecular mechanisms in progressive idiopathic pulmonary fibrosis. Ann. Rev. Med. 64, 265-276.

Szade, K., Zuba-Surma, E., Rutkowski, A.J., Jozkowicz, A., and Dulak, J. (2011). CD45-CD14 +CD34 + murine bone marrow low-adherent mesenchymal primitive cells preserve multilineage differentiation potential in long-term in vitro culture. Mol. Cells 31, 497-507.

Taniguchi, H., Ebina, M., Kondoh, Y., Ogura, T., Azuma, A., Suga, M., Taguchi, Y., Takahashi, H., Nakata, K., Sato, A., et al. (2010). Pirfenidone in idiopathic pulmonary fibrosis. Eur. Respir. J. 35 , 821-829.

Zhao, F., Zhang, Y.F., Liu, Y.G., Zhou, J.J., Li, Z.K., Wu, C.G., and Qi, H.W. (2008). Therapeutic effects of bone marrow-derived mesenchymal stem cells engraftment on bleomycin-induced lung injury in rats. Transplant. Proc. 40, 1700-1705.

Zhao, M.M., Cui, J.Z., Cui, Y., Li, R., Tian, Y.X., Song, S.X., Zhang, J., and Gao, J.L. (2013). Therapeutic effect of exogenous bone marrowderived mesenchymal stem cell transplantation on silicosis via paracrine mechanisms in rats. Mol. Med. Rep. 8 741-746. 